\title{
DISEÑO DE UN MODELO DE EVALUACIÓN DE LA CALIDAD TECNOLÓGICA DE EQUIPOS Y MAQUINARIA
}

\author{
DESIGN OF A MODEL FOR ASSESSING THE TECHNOLOGICAL \\ QUALITY OF EQUIPMENT AND MACHINERY
}

${ }^{1}$ José Mercedes Zuta Rubio

RESUMEN

En este estudio se da a conocer el logro de un Modelo de Evaluación de la Calidad Tecnológica de Equipos y Maquinaria concebido bajo el enfoque sistémico que juzga el valor de los mismos de acuerdo con determinadas variables que promueve la innovación tecnológica del procesamiento industrial como brújula fundamental para mejorar la competitividad de la empresa adquiridora. El modelo de evaluación ha sido desarrollado aplicando el método de aproximaciones sucesivas, partiendo de especificaciones preliminares, hasta satisfacer el objetivo y el juicio experto, en base a datos proporcionados en el marco teórico. El diseño final establece las especificaciones al detalle de los siguiente aspectos estructurales del modelo: 1. focos de valoración, 2. variables a usar en la valoración, 3. Valoración de las variables, 4. datos necesarios en función de los indicadores, 5. fuentes, técnicas e instrumentos para recolectar datos, 6. procedimientos de aplicar la valoración de las variables, 7. análisis de los resultados.

Palabras clave: Calidad, modelo de evaluación, equipamiento, maquinaria.

\section{ABSTRACT}

In this study the achievement of a Model for Evaluation of Technological Quality for Equipment and Machinery is given to know. It was designed assuming the systemic approach that considers their value according to certain variables which promote technological innovation in the industrial processing as fundamental "compass "to improve the competitiveness of the acquiring company. The evaluation model has been developed using the method of successive approximations, based on preliminary specifications, followed by successive improvements, until it satisfies the objective and expert judgment based on information or necessary data provided in the theoretical framework. The final design sets specifications in detail of the following structural aspects of the model: 1 . foci valuation, 2. variables to be used in the valuation, 3. Evaluation of the variables, 4. data necessary based on indicators 5. Sources, techniques and tools to collect data, 6. Procedures for applying the valuation of variables, 7. Analysis of the results.

Keywords: Quality, model for assessing, equipment, machinery.

\section{INTRODUCCIÓN}

Los equipos y maquinaria son uno de los recursos más valiosos donde podemos introducir la innovación tecnológica en el procesamiento industrial. El método de valoración de los equipos y maquinarias es una de las claves para conjugar su adquisición con la estrategia de mejorar la tecnología del proceso industrial. Sin embargo al plantearse el requerimiento de adquisición de equipos y maquinaria, no se encuentra la configuración de un modelo específico para llevar a cabo la valoración con tal enfoque.

Consecuentemente con lo expresado anteriormente uno de los problemas mayores en la adquisición de equipos y maquinaria es cómo juzgar, en un proceso de selección, el valor de la tecnología de las propuestas para contribuir al incremento de la velocidad del desarrollo tecnológico del procesamiento industrial.

Por tanto el objetivo de la investigación fue proponer un modelo de evaluación que juzgue el valor de los equipos y maquinaria de acuerdo con determinadas variables que promuevan la innovación tecnológica como brújula fundamental para mejorar la competitividad de la empresa.

El modelo de evaluación de la calidad tecnológica de equipos y maquinaria, tiene mucha importancia en la medida que señala un procedimiento definido que ayudará a las empresas a tener la capacidad para dirigir y conducir la selección y adquisición de equipos y maquinaria entre propuestas alternativas, siguiendo un proceso riguroso, controlado y sistemático.

\section{MATERIALES Y MÉTODOS}

El método aplicado para el logro del modelo de evaluación ha sido el de diseño de productos, que comprende las fases de concepción y selección, desarrollo y diseño final del modelo, en base a la búsqueda, clasificación e interpretación de la información especializada proporcionados en la referencia bibliográfica. En la concepción se contem- 
Zuta, J. Diseño de un modelo de evaluación de la calidad tecnológica de equipos y maquinaria.

pló las configuraciones de posibles modelos alternativos en respuesta al problema y objetivo de la investigación; en la selección se escogió la alternativa que presentó mayores posibilidades de viabilidad de desarrollo; en el desarrollo se optimizó las especificaciones generales del modelo escogido aplicando el método de ensayo y error; y en el diseño final se estableció las especificaciones al detalle de los aspectos estructurales del modelo. Las fases se han dado iterativamente, con una retroalimentación entre ellas. En la búsqueda de información se utilizó las técnicas de revisión de documentos bibliográficos que incluye la delimitación de temas, búsqueda de las fuentes, selección de la información y de aprehensión de la información. El proceso de diseño se guió por el conocimiento técnico del diseñador.

\section{RESULTADOS Y DISCUSIÓN}

\section{Modelo de evaluación de la calidad tecnológica de equipos y maquinaria}

\subsection{Concepción del modelo de evaluación}

Considerando que los tipos de calidad se diferencian dependiendo de la relación de atributos que lo conforman y del ámbito de aplicación, se ha identificado la calidad tecnológica como un tipo de calidad conformada por los atributos de diseño cuyo perfeccionamiento, en base a la innovación, viene creciendo e irá aumentando en forma continua en el futuro, y que se aplica en el ámbito de la ingeniería. En este contexto, la valoración de la calidad tecnológica en un proceso de selección de equipos y maquinaria permite entregar al cliente o usuario no lo que quiere, sino lo que nunca se había imaginado que quería y que una vez que lo obtenga, se dé cuenta que era lo que siempre había querido

El modelo para evaluar la calidad tecnológica es un prototipo que ha sido concebido asumiendo el enfoque sistémico que juzga el valor de los equipos y máquinas de acuerdo con determinadas variables que promuevan la innovación tecnológica del procesamiento industrial como brújula fundamental para mejorar la competitividad de la empresa adquiridora. Ia medición y análisis de los niveles de cumplimiento de los indicadores, realizada por cl juicio experto de los especialistas, determinan las variables de confiabilidad, funcionalidad, calidad tecnológica y la brecha de calidad tecnológica que distinguen entre máquinas y equipos homólogos (en función de resultados y contexto) cual es el de mejor o peor calidad tecnológica referente al ideal.

Según el enfoque sistémico, la evaluación de la calidad tecnológica de los equipos y maquinaria resulta de la incidencia de la siguiente relación: cualquier innovación en la estructura (insumos) y el funcionamiento (proceso) se proyecta como una influencia beneficiosa o perjudicial en los atributos de la calidad tecnológica (resultado) de equipos y máquinas. $\mathrm{Al}$ respecto, la calidad tecnológica es considerada, cn sentido amplio, como eficaz, de correspondencia entre los propósitos formulados y los logros obtenidos.

\subsection{Estructura del modelo}

El modelo de evaluación aborda los siguientes aspectos estructurales:
1. Elementos o focos de valoración

2. Variables a usar en la evaluación

3. Valoración de las variables

4. Datos necesarios en función de los indicadores

5. Fuente y técnicas para recolectar datos

6. Procedimiento de aplicación en la valoración de las variables

7. Análisis de resultados

\subsection{Especificaciones al detalle de los aspectos estruc- turales del modelo}

\subsubsection{Elementos o focos de valoración}

Siguiendo el enfoque sistémico, se ha identificado los siguientes focos de valoración:

A nivel de insumos

- Los componentes de la estructura de los equipos y máquinas

A nivel de procesos

- La forma de funcionamicnto

A nivel de resultados

- La calidad tecnológica o eficacia de los equipos y maquinaria

\subsubsection{Variables a usaren la evaluacion}

Se ha determinado como variables de evaluación de la calidad tecnológica a las características o atributos de diseño de la estructura y forma de funcionamiento de los equipos y máquinas cuyo perfeccionamiento, en base a la innovación tecnológica, viene creciendo e irá aumentando en forma continua en el futuro.

La característica de diseño de la estructura, de mayor perfeccionamiento continuo en base a la innovación, es la confiabilidad; que a su vez depende de las características de diseño, tales como la calidad de conservación, la simplicidad y la mantenibilidad.

Las características de diseño de la forma de funcionamiento, de mayor perfeccionamiento continuo en base a la innovación, es la funcionalidad; que a su vez depende de otras características de diseño, tales como automatización, rapidez, versatilidad y método de cjecución de la función operativa.

Teniendo en cuenta lo anterior y la operacionalización, las variables para la evaluación de la calidad tecnológica se presentan en la tabla 1, organizadas en forma jerárquica, que consta de dos indices y siete indicadores.

\subsubsection{Valoración de las variables}

\section{A. Valoración de los indicadores}

Para la valoración más adecuada de los indicadores considerados en la tabla 1, se ha elaborado la Tabla 2, escala de valor de rango numérico con la siguiente estructura:

a) El establecimiento de cuatro categorías en orden jerárquico acerca de cuanto se ha logrado entre lo que puede ser considerado deficiente o inadecuado, regular o aceptable, bueno o competente y excelente o sobresaliente.

b) La determinación del rango de puntaje de calificación de la escala de 0 a 7 atendiendo al orden existente entre las categorias, y del rango de puntaje en cada categoría de 1 .

Los rangos de puntaje así establecidos garantizan apreciar que los aspectos de los elementos o focos de valoración de 
Zuta, J. Diseño de un modelo de evaluación de la calidad tecnológica de equipos y maquinaria.

una máquina que estén en una posición ventajosa mínima frente a la de otros, obtengan calificaciones más altos de los indicadores.

c) La definición clara de un patrón de criterios de enjuiciamiento que guardan dependencia con los datos subsumi-

Tabla 1. Variables de evaluación de la calidad tecnológica.

\begin{tabular}{|c|c|c|}
\hline $\begin{array}{c}\text { Variables de mayor } \\
\text { jerarquía }\end{array}$ & Índices & Indicadores \\
\hline \multirow[t]{7}{*}{$\begin{array}{l}\text { Calidad tecnológica } \\
\text { y Brecha }\end{array}$} & 1.Confiabilidad (C) & $\begin{array}{l}\text { 1.1 Calidad de conservación } \\
\text { (Ca) }\end{array}$ \\
\hline & & 1.2 Simplicidad $(\mathrm{Cb})$ \\
\hline & & $\begin{array}{l}\text { 1.3 Mantenibilidad }(\mathrm{Cc}) \\
\text { (seccionamiento, } \\
\text { estandarización) }\end{array}$ \\
\hline & 2. Funcionalidad (F) & 2.1 Rapidez (Fa) \\
\hline & & 2.2 Versatilidad $(\mathrm{Fb})$ \\
\hline & & 2.3 Automatización $(\mathrm{Fc})$ \\
\hline & & $\begin{array}{l}\text { 2.4 Método de ejecución de la } \\
\text { función operativa( }(\mathrm{Ra})\end{array}$ \\
\hline
\end{tabular}

Fuente: Elaboración propia

Tabla 2. Escala de valor para valoración de los Indicadores. Indice: Confiabilidad (C)

\begin{tabular}{|c|c|c|c|}
\hline Indicador & Categoria & Rango de Puntaje & Criterios de Enjuiciamiento \\
\hline $\begin{array}{l}\text { 1.1. Calidad de conservación } \\
\text { (X1) }\end{array}$ & $\begin{array}{l}\text { 1.1.1. Excelente } \\
\text { 1.1.2. Bueno } \\
\text { 1.1.3. Regular } \\
\text { 1.1.4. Deficiente }\end{array}$ & $\begin{array}{l}6-7 \\
4-5 \\
2-3 \\
0-1\end{array}$ & $\begin{array}{l}\text { a) resistencia al desgaste y deterioro. } \\
\text { b) prestigio de la marca. } \\
\text { c) desarrollo industrial del país de origen. } \\
\text { d) desarrollo industrial del país de fabricación. } \\
\text { e) durabilidad. } \\
\text { f) correspondencia del costo. }\end{array}$ \\
\hline 1.2 Simplicidad (Y1) & $\begin{array}{l}\text { 1.2.1. Excelente } \\
\text { 1.2.2. Bueno } \\
\text { 1.2.3. Regular } \\
\text { 1.2.4. Deficiente }\end{array}$ & $\begin{array}{l}6-7 \\
4-5 \\
2-3 \\
0-1\end{array}$ & $\begin{array}{l}\text { a) reducción del espacio que ocupa y peso; } \\
\text { b) facilidad de operar (alivio de los esfuerzos físicos y mentales del operario o } \\
\text { usuario). }\end{array}$ \\
\hline $\begin{array}{l}\text { 1.3. Mantenibilidad (estanda- } \\
\text { rización y seccionamiento) } \\
\text { (Z1) }\end{array}$ & $\begin{array}{l}\text { 1.3.1. Excelente } \\
\text { 1.3.2. Bueno } \\
\text { 1.3.3. Regular } \\
\text { 1.3.4. Deficiente }\end{array}$ & $\begin{array}{l}6-7 \\
4-5 \\
2-3 \\
0-1\end{array}$ & $\begin{array}{l}\text { Criterios de estandarización } \\
\text { a) cercanía de la disponibilidad de repuestos; } \\
\text { b) cercanía de la disponibilidad de servicio técnico; } \\
\text { c) disponibilidad de información técnica } \\
\text { d) actualidad del modelo; } \\
\text { e) alcance nacional o internacional de las normas de fabricación; } \\
\text { f) alcance nacional o internacional de la certificadora; } \\
\text { g) conservación en el mercado; criterios de seccionamiento } \\
\text { h) facilidad para el desmontaje y montaje }\end{array}$ \\
\hline
\end{tabular}

\section{Indice: Funcionalidad $(f)$}

\begin{tabular}{llr}
\hline Indicador & Categoria & Rango de \\
\hline 2.1. Rapidez (X2) & 2.1.1. Excelente & $6-7$ \\
& 2.1.2. Bueno & $4-5$ \\
& 2.1.3. Regular & $2-3$ \\
& 2.1.4. Deficiente & $0-1$ \\
2.2. Versatilidad (Y2) & 2.2.1. Excelente & $6-7$ \\
& 2.2.2. Bueno & $4-5$ \\
& 2.2.3. Regular & $2-3$ \\
& 2.2.4. Deficiente & $0-1$
\end{tabular}

2.3. Automatización $(\mathrm{Z} 2) \quad$ 2.3.1. Excelente 6-7

2.3.2. Bueno 4-5

2.3.3. Regular $2-3$

2.3.4. Deficiente $\quad 0-1$

2.4. Método de ejecución de 2.4.1. Excelente 6-7 la función operativa (W) 2.4.2. Bueno

2.4.3. Aceptable

2.4.4. Inadecuado dos en los indicadores, según se precisa en la figura 1. Los criterios son las bases del enjuiciamiento de lo que se evalúa.

El rango de puntaje de la escala y el rango de puntaje en cada categoría establecidos garantizan apreciar que los aspectos de los focos de valoración de una máquina, que estén en una posición ventajosa mínima frente a la de otros, obtengan calificaciones de los indicadores con puntajes más altos.

El puntaje del indicador es el promedio de las calificaciones de los elementos y el de estos, a su vez, de los aspectos correspondientes, contenidos en la columna de definición de la escala.

\section{B. Valoración de losindices}

\section{i) Valoración delpuntaje de la confiabilidad}

Considerando la organización de las variables de la tabla 1, el puntaje de Confiabilidad (C) es determinado por la distribución del valor máximo de calificación de la confiabilidad (CM) entre los indicadores de calidad de conservación

h) facilidad para el desmontaje y montaje 
Zuta, J. Diseño de un modelo de evaluación de la calidad tecnológica de equipos y maquinaria.

(Ca), simplicidad (Cb) y mantenibilidad (Cc), en función de sus pesos y calificaciones, según la siguiente relación, propuesta por la presente investigación:

$$
\begin{aligned}
\mathrm{C}= & \mathrm{CM} \times \mathrm{PCa}(\mathrm{X} 1 / \mathrm{X} 1 \mathrm{M})+\mathrm{CM} \times \mathrm{PCb}(\mathrm{Y} 1 / \mathrm{Y} 1 \mathrm{M})+\mathrm{CM} \times \\
& \mathrm{PCc}(\mathrm{Z} 1 / \mathrm{Z} 1 \mathrm{M})
\end{aligned}
$$

Donde:

C: Puntaje de valoración del indicador confiabilidad.

CM: Puntaje máximo de calificación convenido de $\mathrm{C}$.

PCa, PCb, PCc: Pesos de los indicadores $\mathrm{Ca}, \mathrm{Cb}, \mathrm{Cc}$, respectivamente.

$\mathrm{X} 1, \mathrm{Y} 1, \mathrm{Z} 1$ : Son las calificaciones de los indicadores $\mathrm{Ca}, \mathrm{Cb}$, Cc, respectivamente.

X1M, Y1M, Z1M: Son las calificaciones máximas, según escala, de los indicadores $\mathrm{Ca}, \mathrm{Cb}, \mathrm{Cc}$, respectivamente.

Para:

$$
\begin{array}{ll}
\mathrm{PCa}=0,4 & \mathrm{CM}=25 \\
\mathrm{PCb}=0,2 & \mathrm{X} 1 \mathrm{M}=7 \\
\mathrm{PCc}=0,4 & \mathrm{Y} 1 \mathrm{M}=7 \\
& \mathrm{Z} 1 \mathrm{M}=7
\end{array}
$$

La fórmula simplificada de $\mathrm{C}$ es:

$$
\begin{aligned}
& \mathrm{C}=0,4 \times 25(\mathrm{X} 1 / 7)+0,2 \times 25(\mathrm{Y} 1 / 7)+0,4 \times 25(\mathrm{Z} 1 / 7) \\
& \mathrm{C}=\underline{10 \times 1+5 \mathrm{Y} 1+10 \mathrm{Z} 1}
\end{aligned}
$$

La asignación de la ponderación o peso de 0,4 a PCa y PCc obedece a que los indicadores calidad de conservación (Ca) y mantenibilidad (Cb) son los de mayor importancia relativa pues constituyen la razón de ser de cualquier equipo o máquina; el otro indicador simplicidad es complementario de los anteriores, pero no por ello de carácter superfluo.

La asignación de 25 puntos al puntaje máximo del indicador confiabilidad (CM) obedece a la distribución del puntaje de la calidad de máquina.

La asignación de 7 puntos a X1M, Y1M, Z1M, calificaciones máximas de los indicadores $\mathrm{Ca}, \mathrm{Cb}$ y $\mathrm{Cc}$, obedece a lo determinado para la escala de valor, y garantiza que quien cumpla puede estar en una posición ventajosa, frente a otro, en el índice confiabilidad.

\section{ii) Valoración del puntaje de la funcionalidad}

Considerando la organización de las variables de la tabla 1, el Puntaje de la funcionalidad $(\mathrm{F})$ es determinado por la distribución del valor máximo de calificación de la funcionalidad (FM) entre los indicadores: rapidez del proceso $(\mathrm{Fa})$, versatilidad del proceso $(\mathrm{Fb})$, automatización del proceso (Fc) y método de ejecución de la función operativa del proceso (Ra), en función de sus pesos y calificaciones, según la siguiente relación:

\section{$\mathrm{F}=\mathrm{FM} \times \mathrm{PFa}(\mathrm{X} 2 / \mathrm{X} 2 \mathrm{M})+\mathrm{FM} \times \mathrm{PFb}(\mathrm{Y} 2 / \mathrm{Y} 2 \mathrm{M})+\mathrm{FM} \times$ $\mathrm{PFc}(\mathrm{Z} 2 / \mathrm{Z} 2 \mathrm{M})+\mathrm{FM} \times \mathrm{PRa}(\mathrm{W} / \mathrm{WM})$}

Donde:

F: Puntaje de calificación de la variable de la funcionalidad. $\mathrm{PFa}, \mathrm{PFb}, \mathrm{PFc}, \mathrm{PRa}$ : Son los pesos de los indicadores $\mathrm{Fa}, \mathrm{Fb}$, $\mathrm{Fc}, \mathrm{Ra}$
FM: Puntaje máximo de calificación convenido de F X2, Y2, Z2, W: Son las calificaciones, scgún escala de valoración, de los indicadores $\mathrm{Fa}, \mathrm{Fb}, \mathrm{Fc}$, Ra, respectivamente. X2M, Y2M, Z2M, WM: Son las calificaciones máximas, según escala de valoración, de los indicadores $\mathrm{Fa}, \mathrm{Fb}, \mathrm{Fc}, \mathrm{Ra}$.

Para:

$$
\begin{array}{ll}
\mathrm{PFa}=0,2 & \mathrm{FM}=25 \\
\mathrm{PFb}=0,2 & \mathrm{X} 2 \mathrm{M}=7 \\
\mathrm{PFc}=0,1 & \mathrm{Y} 2 \mathrm{M}=7 \\
\mathrm{PRa}=0,5 & \mathrm{Z} 2 \mathrm{M}=7 \\
& \mathrm{WM}=7
\end{array}
$$

La fórmula simplificada de F es:

$$
\begin{aligned}
\mathrm{F}= & 0,2(25)(\mathrm{X} 2 / 7)+0,2 \times 25(\mathrm{Y} 2 / 7)+0,1 \times 25(\mathrm{Z} 2 / 7)+0,5 \\
& \times 25(\mathrm{~W} / 7) \\
\mathrm{F}= & \frac{5 \mathrm{X} 2+5 \mathrm{Y} 2+2,5 \mathrm{Z} 2+12,5 \mathrm{~W}}{7}
\end{aligned}
$$

La asignación del peso de 0,5 mayor a PRa obedece a que el indicador método de ejecución de la función operativa (Ra) es el de mayor importancia relativa pues constituyen la razón de ser de cualquier máquina; los otros indicadores: velocidad de operación, versatilidad y automatización son complementarios del anterior, pero no por ello de carácter superfluo.

La asignación de 25 puntos al puntaje máximo del indice funcionalidad (FM) obedece a la distribución del puntaje de la calidad de la máquina.

La asignación de 7 puntos a X2M, Y2M, Z2M, WM, calificaciones máximas de los sub-indicadores $\mathrm{Fa}, \mathrm{Fb}, \mathrm{Fc}$ y $\mathrm{Ra}$, obedece a lo determinado para la escala de valor, y garantiza que quien cumpla puede estar en una posición ventajosa, frente a otro, en el indicador funcionalidad.

\section{iii) Valoración del puntaje de calidad tecnológica}

Considerando la organización de las variables de la tabla 1, el Puntaje de calidad (PTi) es determinado por la distribución de puntajes de la valoración máxima de la calidad tecnológica (PTM), entre los índices confiabilidad (C) y funcionalidad (F), en función de sus pesos y valoraciones, según la siguiente relación:

\section{$\mathrm{PT} i=\mathrm{PTM} \times \mathrm{PC} \times \mathrm{C} / \mathrm{CM}+\mathrm{PTM} \times \mathrm{PF} \times \mathrm{F} / \mathrm{FM}$}

PTi: Puntaje de calidad de la máquina $\mathrm{i}$

PC, PF: Peso de los indicadores confiabilidad y funcionalidad, respectivamente.

PTM: Puntaje de valoración máxima de la calidad de la máquina.

C, F: Puntajes obtenidos de las variables confiabilidad y funcionalidad, respectivamente.

CM, FM: Puntajes máximos de los indices confiabilidad y funcionalidad, respectivamente.

Para:

$\begin{array}{ll}\mathrm{PC}=0,4 & \mathrm{PTM}=50 \\ \mathrm{PF}=0,6 & \mathrm{CM}=25 \\ & \mathrm{FM}=25\end{array}$


Zuta, J. Diseño de un modelo de evaluación de la calidad tecnológica de equipos y maquinaria.

La fórmula simplificada de PTies:

$\mathrm{PTi}=0,4 \times 50 \times \mathrm{C} / 25+0,6 \times 50 \times \mathrm{R} / 25$

$\mathrm{PTi}=\underline{20 \mathrm{C}+30 \mathrm{~F}}$

25

La asignación de un peso mayor a $\mathrm{PF}(0,6)$ obedece a que el indicador funcionalidad (F) es el de mayor importancia relativa que el indicador confiabilidad $(\mathrm{C})$, que es complementario del anterior pero no por ello de primera importancia.

La asignación de 50 puntos al puntaje máximo de calificación de la calidad de la máquina (PTM) y de 25 puntos a los puntajes máximos de calificación de índices de confia- bilidad (CM) y funcionalidad (FM) son valores convenidos para garantizar que quien cumpla con puntajes más altos cstć en una posición ventajosa frente a la de otros equipos y maquinarias.

\section{iv) Valoración de la brecha de la calidad tecnológica}

La brecha o desfase de la calidad es consecuencia del incremento del valor agregado que genera la innovación. La innovación modifica la máquina con la aplicación por primera vez de conocimientos en los elementos o focos de valoración, con el objeto de mejorar su función y hacerlo más competitivo.

La valoración de la brecha o desfase es estimado restando del puntaje máximo de valoración de la calidad

Formulario 1: Para recolectar datos referidos a los indicadores Máquina:

Fecha:

\begin{tabular}{|c|c|}
\hline $\begin{array}{l}\text { Datos relevantes referidos a los Indicadores } \\
\text { de la calidad tecnológica }\end{array}$ & $\begin{array}{l}\text { Información correspondiente a los componentes de } \\
\qquad \begin{array}{l}\text { los equipos y máquinas } \\
\text { 1. mecanismo operador, 2.piezas de complementación operacional } \\
\text { 3.piczas auxiliares, 4.mecanismos de accionamiento, 5.carcasa }\end{array}\end{array}$ \\
\hline \multicolumn{2}{|l|}{ Îndice: Confiabilidad de la estructura } \\
\hline \multicolumn{2}{|l|}{ Calidad de Conservación (X1) } \\
\hline \multicolumn{2}{|l|}{ a) Materiales de fabricación (principalmente de las partes críticas de falla y deterioro) } \\
\hline \multicolumn{2}{|l|}{ b) Marca o Fabricante (\% de las partes según marca) } \\
\hline \multicolumn{2}{|l|}{ c) País de origen (\% de las partes según origen) } \\
\hline \multicolumn{2}{|l|}{ d) País de fabricación de las partes, made in (\% de las partes construidas por el país de origen) } \\
\hline \multicolumn{2}{|l|}{$\begin{array}{l}\text { e) Vida útil estimada por el fabricante } \\
\text { Garantia de funcionamiento }\end{array}$} \\
\hline \multicolumn{2}{|l|}{ f) Costo de adquisición } \\
\hline \multicolumn{2}{|l|}{ Simplicidad (Y1) } \\
\hline \multicolumn{2}{|l|}{ a) Dimensiones $(1 \times a \times h)$} \\
\hline \multicolumn{2}{|l|}{ b) Tipo de transmisión del movimiento } \\
\hline \multicolumn{2}{|l|}{ Mantenibilidad (Z1) } \\
\hline \multicolumn{2}{|l|}{ Datos de Estandarización } \\
\hline \multicolumn{2}{|l|}{ a) Lugar más próximo de punto de venta de repuestos } \\
\hline \multicolumn{2}{|l|}{ b) Lugar más próximo de suministro o procedencia del servicio técnico } \\
\hline \multicolumn{2}{|l|}{ c) Disponibles de fuentes escritas que proveen información técnica de la máquina } \\
\hline \multicolumn{2}{|l|}{$\begin{array}{l}\text { d) Modelo } \\
\text { Año de fabricación }\end{array}$} \\
\hline \multicolumn{2}{|l|}{ e) Normas de fabricación } \\
\hline \multicolumn{2}{|l|}{ f) Certificadora de la calidad de cumplimiento de las normas de fabricación. } \\
\hline \multicolumn{2}{|l|}{ g) Empresas en las que se encuentra en uso la máquina por años de venta. } \\
\hline \multicolumn{2}{|l|}{ Datos de Seccionamiento } \\
\hline \multicolumn{2}{|l|}{ h) Tipos de unión y ubicación de las partes críticas de falla y deterioro } \\
\hline \multicolumn{2}{|l|}{ İndice: Funcionalidad del proceso } \\
\hline \multicolumn{2}{|l|}{ Rapidez de Operación (X2) } \\
\hline \multicolumn{2}{|l|}{$\begin{array}{l}\text { a) Tiempo del ciclo de la operación } \\
\text { Tipo de flujo }\end{array}$} \\
\hline \multicolumn{2}{|l|}{ Versatilidad (Y2) } \\
\hline \multicolumn{2}{|l|}{ a) Rangos de variación de la capacidad de producción } \\
\hline \multicolumn{2}{|l|}{$\begin{array}{l}\text { b) Rangos de variación de los parámetros de operación (dimensiones, velocidades, temperatura, } \\
\text { tiempo, presión, etc.) }\end{array}$} \\
\hline \multicolumn{2}{|l|}{ c) Tipo de interconexión con otras máquinas (rígida o flexible o no conectable) } \\
\hline d)Tipos de los elementos en proceso a la entrada (tamaños, composición, característica) & \\
\hline e) Tipos de elementos en proceso a la salida (tamaños, composición, característica) & \\
\hline Automatización (Z2) & \\
\hline a) Tipos de instrumentos de control y regulación. & \\
\hline b) Requerimiento de mano de obra directa y calificación & \\
\hline Método de (W) ejecución de la función operativa & \\
\hline a) Principio y procedimiento de ejecución de la función operativa & \\
\hline b) Cantidad del consumo de energía, otra o ambas. & \\
\hline $\begin{array}{l}\text { c) Dispositivos de tratamiento o canalización de residuos sólidos, líquidos y vapores. } \\
\text { Ruido en decibeles que se producen al funcionar. } \\
\text { Forma estética }\end{array}$ & \\
\hline
\end{tabular}

Figura 1. Modelo de Formulario para recolectar información.

Fuente: Elaboración propia 
Zuta, J. Diseño de un modelo de evaluación de la calidad tecnológica de equipos y maquinaria.

tecnológica (PTM), puntaje logrado por el programa académico en estudio (PTi), según la siguiente fórmula:

$$
\mathrm{BT}=\mathrm{PTM}-\mathrm{PTi}
$$

\subsubsection{Datos necesarios en función de los indicadores}

La identificación y construcción de datos capaces de aportar información relevante para valorar los indicadores de evaluación han sido realizados en base a las definiciones de los índices e indicadores y buscando dar respuesta a los criterios de enjuiciamiento de la escala de valor, los mismos que se dan en el Formulario 1 mostrado en la figura 1.

\subsubsection{Fuente y técnicas para recolectar datos}

Para la recogida de datos necesarios, mediante la técnica de observación directa, se tiene como fuente a las mismas máquinas.

Para la recogida de datos, mediante la técnica de revisión de documentos, se tiene como fuentes los documentos escritos, cifrados e iconográficos del fabricante de las máquinas (manuales de operación, procedimientos estándares de operación, catálogos y folletos de los equipos y maquinaria). Para la recogida de datos necesarios, mediante la técnica de interrogación, se tiene como fuentes a los proveedores, fabricantes o personas expertas.

Tabla 3.Valoración de los indicadores de una máquina por cada dato.

\begin{tabular}{|c|c|c|c|c|c|c|c|}
\hline \multicolumn{3}{|c|}{ Formato de valoración $\mathrm{N}^{\circ}: 1$} & \multicolumn{3}{|c|}{ Fecha: } & & \\
\hline \multirow{2}{*}{$\begin{array}{l}\text { Datos por } \\
\text { indicador }\end{array}$} & \multicolumn{7}{|c|}{ Puntajes de valoración de los indicadores por datos } \\
\hline & $\mathrm{X} 1$ & $\mathrm{Y} 1$ & $\mathrm{Z1}$ & $\mathrm{X} 2$ & $\mathrm{Y} 2$ & $\mathrm{Z2}$ & W \\
\hline \multicolumn{8}{|l|}{ a) } \\
\hline \multicolumn{8}{|l|}{ b) } \\
\hline \multicolumn{8}{|l|}{ c) } \\
\hline \multicolumn{8}{|l|}{ d) } \\
\hline \multicolumn{8}{|l|}{ c) } \\
\hline \multicolumn{8}{|l|}{ f) } \\
\hline \multicolumn{8}{|l|}{ g) } \\
\hline \multicolumn{8}{|l|}{ h) } \\
\hline \multicolumn{8}{|l|}{ i) } \\
\hline \multicolumn{8}{|l|}{ i) } \\
\hline \multicolumn{8}{|l|}{ k) } \\
\hline \multicolumn{8}{|l|}{ 1) } \\
\hline Puntaje medio & & & & & & & \\
\hline
\end{tabular}

Fuente: Elaboración propia

Dónde: $\mathrm{X} 1$ calidad de conservación, $\mathrm{Y} 1$ simplicidad, $\mathrm{Z} 1$ mantenibilidad, $\mathrm{X} 2$ velocidad, $\mathrm{Y} 2$ versatilidad, $Z 2$ automatización, $\mathrm{W}$ eficacia

Tabla 4. Valoración promedio de los Indicadores de las máquinas.

\begin{tabular}{|c|c|c|c|c|c|c|c|}
\hline Formato de valoración $N$ & & & & echa & & & \\
\hline Marcas de las máquinas & & tajes & & $\begin{array}{l}\text { o de } \\
\text { icade }\end{array}$ & & & \\
\hline & $\mathrm{X} 1$ & Y1 & $\mathrm{Z1}$ & $\mathrm{X} 2$ & $\mathrm{Y} 2$ & $\mathrm{Z} 2$ & w \\
\hline
\end{tabular}

3

4

Fuente: Elaboración propia

Dónde: X1 calidad de conscrvación, Y1 simplicidad, Z1 mantenibilidad, $\mathrm{X} 2$ rapidez, $\mathrm{Y} 2$ versatilidad, $\mathrm{Z} 2$ automatización, $\mathrm{W}$ método operativo
3.3.6. Procedimiento de aplicación en la valoración de las variables El procedimiento de aplicación en la valoración de las variables comprende:

\section{(1) La valoración de los indicadores}

La valoración de los indicadores es realizada, por el juicio experto de los especialistas, por conversión, confrontando los criterios de enjuiciamiento establecidos en la escala de valor de la tabla 2 con los datos recolectados en el Formulario 1, por cada indicador, hasta ubicar su categoría relativa y puntaje. Los puntajes de los indicadores obtenidas por cada dato y el promedio de datos son vaciados en las tablas 3 y 4 siguientes, respectivamente.

Los datos recopilados y organizados por indicadores, permiten valorar los indicadores con puntajes interpretables y comparables.

\section{(2) La valoración de los indices de confiabilidad (C) y funcionalidad (F)}

La valoración de los indices de Confiabilidad (C) y Funcionalidad $(\mathrm{F})$ de las máquinas es realizada reemplazando los valores promedio obtenidos de los indicadores correspondientes en las fórmulas (1) y (2):

$\mathrm{C}=\left(10 \mathrm{X}_{1}+5 \mathrm{Y}_{1}+10 \mathrm{Z}_{1}\right) / 7$
$\mathrm{~F}=\left(5 \mathrm{X}_{2}+5 \mathrm{Y}_{2}+2,5 \mathrm{Z}_{2}+12,5 \mathrm{~W}\right) / 7$

Los puntajes de la calificación obtenidos en cada caso son vaciados en la tabla 5 .

\section{(3) La valoración de la calidad tecnológica (PT)}

La valoración de la calidad tecnológica (PT) de las máquinas en evaluación se realiza reemplazando en la fórmula (3), del autor, los valores obtenidos de los indicadores confiabilidad $y$ funcionalidad siguiente:

$\mathrm{Pti}=\underline{20 \mathrm{C}+30 \mathrm{~F}}$

25

\section{(4) I a valoración de la brecha de calidad tecnológica $(B C T)$}

La calificación de la Brecha de calidad tecnológica (BT) de las máquinas en evaluación es realizada reemplazando en la fórmula (4), del autor, los valores del puntaje de calidad máximo PTM de 50 y el puntaje de calidad logrado PTi.

$\mathrm{BT}=\mathrm{PTM}-\mathrm{PTi}$

Los puntajes resultantes de las valoraciones obtenidas de la calidad y de la brecha de calidad tecnológica son vaciados en la tabla 6 .

Tabla 5. Valoración de la Confiabilidad y Funcionalidad de las máquinas.

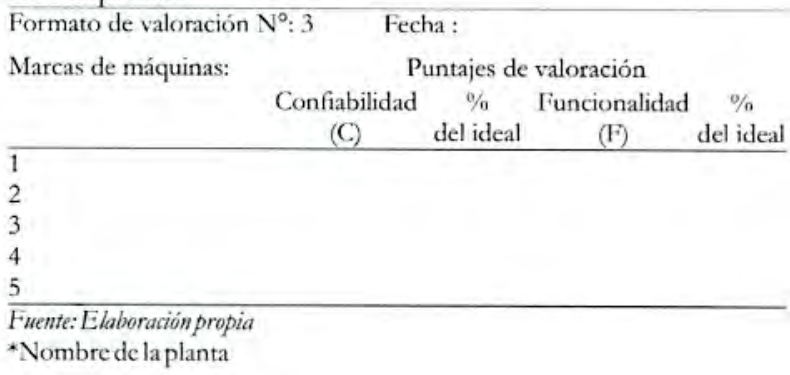


Zuta, J. Diseño de un modelo de evaluación de la calidad tecnológica de equipos y maquinaria.

Tabla 6. Valoración de la calidad y brecha de la calidad tecnológica.

\begin{tabular}{|c|c|c|}
\hline \multicolumn{3}{|c|}{ Formato de valoración $\mathrm{N}^{\circ}: 4$ Fecha: } \\
\hline Marcas de las máquinas & $\begin{array}{c}\text { Calidad } \\
\text { (P') }\end{array}$ & $\begin{array}{c}\text { Brecha } \\
\text { (BT) }\end{array}$ \\
\hline \multicolumn{3}{|c|}{$x^{2}=$} \\
\hline \multicolumn{3}{|l|}{2} \\
\hline \multicolumn{3}{|l|}{3} \\
\hline \multicolumn{3}{|l|}{4} \\
\hline 5 & & \\
\hline
\end{tabular}

\subsubsection{Análisis de los resultados}

Los promedios de los puntajes de valoración obtenidas de la Confiabilidad, Funcionalidad, Calidad y Brecha de la calidad tecnológica de cada máquina en evaluación son confrontados en una matriz de resultados con los puntajes de sus homólogos y puntajes máximos o ideales de valoración, cuyo formato se muestra en la tabla 7 .

$\mathrm{El}$ análisis busca identificar, en un proceso de selección, la máquina que ha obtenido los puntajes de calificación más próximos a los puntajes ideales para recomendar su adquisición; o identificar, en un proceso de evaluación de una línea de producción ya establecida, la máquina que ha obtenido los más bajos puntajes de calificación con el objeto de recomendar su retiro de uso por obsoleta. La tabla 7 presenta la matriz de resultados de las valoraciones.

Tabla 7. Matriz de resultados de las valoración de las máquinas.

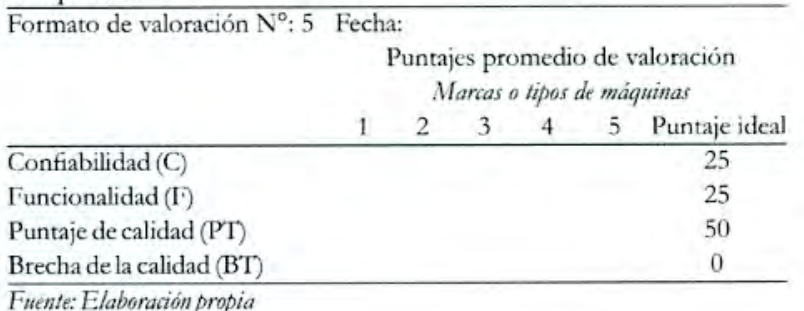

\section{CONCLUSIONES}

1. El modelo de proceso de evaluación ha demostrado su validez a través del juicio de expertos y ha resistido el criterio de la práctica con la aplicación para valorar los equipos y maquinaria de las plantas de procesamiento pesquero.

2. El modelo de proceso de evaluación resulta novedoso y de gran valor por:

- Valorar la calidad tecnológica de los equipos y maquinaria con variables que propician el perfeccionamiento continuo, en base a la innovación y la competitividad.

- Identificar el estado real, las brechas de nivel de calidad tecnológica referente al estado deseado, y las diferencias con equipos y maquinaria homólogos.

- Permitir hacer apreciaciones especificas centrales y hacer apreciaciones generales significativas, coherentes, pertinentes de los equipos y maquinaria.

3. La lógica y consistencia de la estructura de las variables, de la escala y procedimientos de valoración con el objetivo; la especificidad de las definiciones de las variables; los datos de hechos y la participación de expertos hacen confiables y válidos los resultados de la evaluación.

4. El reducido número de las variables y la posibilidad para la obtención de sus valores facilitan la ejecución de los proceso de evaluación

5. La configuración de la matriz de resultados para el análisis permite identificar el orden de la calidad tecnológica de las máquinas, para seleccionar la mejor.

\section{REFERENCIAS BIBLIOGRÁFICAS}

Bennett, D. J., Vaidya, K. G., Zhao, H. y Wang, X. M. (1997). Technology transfer to the China machine tool industry: The need for a technology valuation model. Industry and Higher Education, $\mathrm{Vol} 11, \mathrm{~N}^{\circ} 1$.

Crisologo, A. (1975). Tecnología educativa. Lima - Perú: Editorial Ediciones Abedul, 1996.

Chupakin, V. (1999). Fish - Processing equipment, Moscú: Publicaciones MIR, Primera Edición 1955.

Dobrovoski, V., Zablonski, K., Radchik, A., Erlij, L. (1980). Elementos de máquina, Moscú: Editorial MIR.

FAO. (1995). La selección, prueba y evaluación de máquinas y equipos agrícolas. Servicios agrícolas de la FAO, Boletín.

Fritz, R. (1997). Corrientes corporativas, México: Ediciones Castillo.

Hidalgo, M. (1981). Componentes del sistema de evaluación educacional, Lima: Edición CIPAC, Primera Edición.

Palfrman, A. (1994). Guide fisheries sector studies, FAO, Roma.

Part, S. (1995). Monitoreo y evaluación de impacto para proyectos de desarrollo, Lima: Editorial Galois, Primera Edición.

Phi Delta Kappa. (1971). National study commytion evaluation, Peacock: Itaska, III.

Roger, J. H. (1996). Ponga la calidad a prueba, México: Mc Graw-Hill, Primera Edición.

Rothery, B. (1997). ISO 14000 ISO 9000, México: Panorama Editorial S.A., Primera Edición.

Senn, J. (1992). Análisis y diseño de sistemas de información, México: Editorial Mc. Graw Hill, Interamericana de México, S.A.de C.U.

Tenbrink, T. (1981). Evaluación. Madrid: Ed. NACEA.

Valdés, L. (1959). Innovación, Colombia: Editorial Norma S.A, 2004 Vilbrandt \& Dryden. Chemical engineering plant design, New York: Mc Graw - Hill Book Company, Inc. Fourth Edition.

Vilbrandt F. (1959). Chemical engineering plant design, New York: Mc Graw - Hill Book Company, Inc. Fourth Edition.

Vilbrandt, D. (1957). Chemical enginnering plant design, Tokyo: Editorial Mc Graw - Hill Bork Company, Inc.

VIVIENDA. (1983). Reglamento general de tasaciones del Perú, Resolución Ministerial del Sector de Vivienda y Construcción. Lima: Editorial Hozlo SCRL.

Waller, J. (1997). El manual de administración de la calidad, México: Panorama Editorial S.A., Primera Edición, Tercera Impresión.

Zuta, J. (2000). Diseño de un modelo metodológico de selección de maquinaria y equipo para la industria pesque- 


\section{Ciencia \& Desarrollo}

Zuta, J. Diseño de un modelo de evaluación de la calidad tecnológica de equipos y maquinaria.

ra - Callao - Perú: UNAC.

Zuta, J. (1990). Líneas de procesamiento de pescado y mariscos. Callao-Perú: Editorial UNAC.

Zuta, J. (2002). Evaluación técnica de las fábricas de harina y aceite de pescado. Inédito: Universidad Nacional del Callao.

Zuta, J. (2003). Evaluación tecnológica de maquinaria y equipos de las plantas de conservas de pescado.
Universidad Nacional del Callao.

Zuta, J. (2011). Evaluación tecnológica de maquinaria y equipos de las plantas de congelado de pescado. Universidad Nacional del Callao.

Zuta, J. (2013). Calidad tecnológica de maquinaria y equipos de las plantas de pastas y embutidos de pescado. Universidad Nacional del Callao.

\section{Correspondencia:}

José Mercedes Zuta Rubio: jzutarubio@hotmail.com
Fecha de Recepción: 28/12/2014

Fecha de Aceptación: 30/04/2015 